\title{
TOPOLOGY OF LEVEL CURVES OF HARMONIC FUNCTIONS
}

\author{
BY \\ WILFRED KAPLAN
}

\section{INTRODUCTION}

1.1. Statement of results. The main purpose of this paper is to establish the following theorem.

THEOREM 1. Let $F$ be a regular curve-family filling the $x y$-plane. There exists $a$ homeomorphism of the $x y$-plane onto a domain $D$ of the $x y$-plane such that $F$ is transformed onto the family of level curves of a function $u(x, y)$ which is harmonic in $D$.

By a regular curve-family $\left({ }^{1}\right)$ is meant a family which is locally homeomorphic to a family of parallel lines.

It follows from the theory of conformal mapping that $D$ can always be chosen to be one of the two domains $D_{1}$ : the interior of the unit circle $x^{2}+y^{2}$ $=1, D_{\infty}$ : the entire $x y$-plane. If $D$ can be chosen as $D_{1}, F$ will be termed hyperbolic; if $D$ can be chosen as $D_{\infty}, F$ will be termed parabolic. The cases are not mutually exclusive, as the following theorem shows.

THEOREM 3. Every $F$ is hyperbolic. There exist infinitely many topological types of families $F$ which are not parabolic.

A third result concerns the Riemann surface of the inverse of a function $\phi(z)$ analytic in a simply-connected domain $D$. By the Riemann surface of the inverse of such a function will be meant that part of the Riemann surface of the complete inverse function which corresponds to $D:$ that is, the space of pairs $(z, w), w=\phi(z), z$ in $D$, with local coordinates defined in the usual manner. This would coincide with the Riemann surface of the complete inverse function only if $\phi(z)$ cannot be continued analytically beyond $D$.

TheOREM 2. Let $w=\phi(z)$ be analytic in the simply-connected domain $D$ and have nonvanishing derivative in $D$. Let $R$ be the Riemann surface of the inverse function. Then there exists an at most countably infinite class $A$ and a subdivision of $R$ into nonoverlapping simply-connected subsets $R_{\alpha}(\alpha \in A)$, such that the following conditions are satisfied:

(a) Each set $R_{\alpha}$ is schlicht over the w-plane.

(b) The common boundary points of two sets $R_{\alpha_{1}}$ and $R_{\alpha_{2}}$, if nonvoid, form an open curve, which lies schlicht over a straight line $\operatorname{Re}(w)=$ const. in the w-plane, and which separates $R$.

Presented to the Society, April 25, 1947; received by the editors February 13, 1947.

(1) See [1]. Numbers in brackets refer to the bibliography at the end of the paper. 
(c) The part $W_{\alpha}$ of the w-plane over which $R_{\alpha}$ lies can be represented in the form: $p_{\alpha}(u)<v<q_{\alpha}(u), u \in I_{\alpha}$, whereby the following conditions hold:

(c1) For one value of $\alpha, I_{\alpha}$ is an open (perhaps infinite) interval; for all other values of $\alpha, I_{\alpha}$ is a half-open (perhaps infinite) interval;

(c2) $-\infty \leqq p_{\alpha}(u)<q_{\alpha}(u) \leqq+\infty$;

(c3) $p_{\alpha}(u)$ is upper semi-continuous: $q_{\alpha}(u)$ is lower semi-continuous.

1.2. Relation to previous results. In previous papers [1] [2] the author has classified topologically all regular curve-families filling the plane. It was shown, in particular, that each curve of the family must be open, that is, homeomorphic to an open interval, and must have the point at infinity as unique limit in both directions on the curve. Each family $F$ was shown to be capable of a "normal" subdivision into nonoverlapping subfamilies $F_{\alpha}$, each of which is homeomorphic to a family of parallel lines. (Cf. $\$ 3$ below for a more detailed description.) It is this normal subdivision which gives rise to the subdivision of the Riemann surface $R$ in Theorem 2 .

In addition, the following theorem was established [1, Theorem 42]:

THEOREM A. Every regular curve-family filling the $x y$-plane is the level-curve amily of a continuous function $f(x, y)$ which has no local extrema.

Theorem 1, therefore, states that, after a suitable homeomorphism of the plane has been applied, $f(x, y)$ can actually be chosen as a harmonic function. Conversely, the level-curve family of a function $u(x, y)$ harmonic in a simplyconnected domain $D$ is a regular curve-family filling $D$ (hence a family homeomorphic to a family $F$ filling the plane) provided $u$ has no critical points in $D$. The points at which the partial derivatives $\partial u / \partial x$ and $\partial u / \partial y$ vanish are necessarily singular points of the family (of "multiple saddle-point" type). The result can therefore also be interpreted as achieving a topological characterization and classification of the level-curve families of functions $u(x, y)$ harmonic without critical points in a simply-connected domain $D$.

In another paper [4] the author stated and outlined the proof of the following theorem.

THEOREM B. Every regular curve-family filling the plane can be mapped homeomorphically onto the family of solutions of a system of differential equations

$$
\frac{d x}{d t}=p(x, y), \quad \frac{d y}{d t}=q(x, y), \quad p^{2}+q^{2} \neq 0,
$$

where $p$ and $q$ are defined and have continuous first partial derivatives for all $(x, y)$.

This theorem is a simple consequence of Theorem 1, for the level-curves of the function $u$ satisfy differential equations of the form indicated, with 
$p=\partial u / \partial y$ and $q=-\partial u / \partial x$, and a family defined by (1) in any simply-connected domain is necessarily homeomorphic to a family of same type filling the plane. However, by the method of proof to be employed here, this result will be used in the demonstration of Theorem 1, so that it cannot be considered as a corollary. An alternate method of proof of Theorem 1, independent of Theorem B, will be indicated in $\$ 5$ below.

\section{Proof of Theorem 1}

2.1. Preliminary definitions. Let $F_{1}$ and $F_{2}$ be regular curve-families filling surfaces $S_{1}$ and $S_{2}$. If there is a homeomorphism $T$ of $S_{1}$ onto $S_{2}$ such that $F_{1}$ is transformed onto $F_{2}, F_{1}$ will be termed equivalent to $F_{2}$. If further $S_{1}$ and $S_{2}$ are oriented and $T$ preserves orientation, then $T$ will be called an o-homeomorphism and $F_{1}$ will be termed o-equivalent to $F_{2}$.

Throughout the following the same symbol, for example $F$, will be used to represent a curve-family and the point set which it fills. The context will prevent ambiguity.

For convenience complex variables $z=x+i y$ and $w=u+i v$ will be used to represent the points of the $x y$-plane and the $u v$-plane. Both planes will be assumed oriented in the usual manner, so that an analytic homeomorphism $w=\phi(z)$ is orientation-preserving.

2.2. Construction of the mapping $T_{1}$. From this point on $F$ will be assumed to be defined by differential equations of form (1). By Theorem B, this involves no loss of generality. $G$ will denote the family of solutions of the differential equations

$$
\frac{d x}{d t}=-q(x, y), \quad \frac{d y}{d t}=p(x, y),
$$

that is, the family of orthogonal trajectories of $F . G$ is then also a regular curve-family filling the $x y$-plane [1, Theorem 2].

By Theorem A, continuous functions $f(x, y)$ and $g(x, y)$ can be chosen which have no local extrema and which have $F$ and $G$, respectively, as levelcurve families. These functions will now be assumed chosen in a fixed manner.

The transformation $u=f(x, y), v=g(x, y)$ of the $z$-plane into the $w$-plane will be denoted by $T_{1}$.

LEMma. $T_{1}$ is continuous and is locally a homeomorphism which is either throughout orientation-preserving or throughout orientation-reversing.

Proof. Since the curves of $G$ are orthogonal trajectories of $F$, a neighborhood $U$ of each point $\left(x_{0}, y_{0}\right)$ can be found which can be mapped $o$-homeomorphically on a rectangle $a<x<b, c<y<d$ in such a way that the curves of $F$ become the lines $x=$ constant and the curves $G$ become the lines $y=$ constant of the rectangle. The functions $f$ and $g$ become continuous functions of $x$ and $y$ respectively in the rectangle, and necessarily monotone functions, since 
both functions have no relative extrema. The mapping from the rectangle to the $w$-plane is hence one-to-one and continuous, and the inverse is also continuous. Thus $T_{1}$ is a homeomorphism on $U$, and must either preserve or reverse orientation in $U$. It follows that the set of points for which $T_{1}$ preserves (or reverses) orientation is open. Since the $z$-plane is connected, $T_{1}$ must therefore either throughout preserve or throughout reverse orientation.

By replacing $g$ by $-g$, if necessary, it is possible to ensure that $T_{1}$ preserves orientation and it will be assumed that this has been done.

2.3. Definition of the Riemann surface $R$. A Riemann surface is by definition a surface with local conformal coordinates. The surface $R$ is defined as follows. Its points are all pairs $P:(z, w)$, where $w=T_{1} z$. The mapping $T_{2}: z$ $\rightarrow P:(z, w)$ is thus a one-to-one correspondence between the $z$-plane and $R$ and can be used to define a topology and an orientation in $R$, so that $T_{2}$ becomes an $o$-homeomorphism of $D_{\infty}$ onto $R$. The mapping $T_{3}: P:(z, w) \rightarrow w$ is then a continuous map of $R$ into the w-plane which is locally a homeomorphism. This allows one to define local conformal coordinates in $R$. For a neighborhood of each $P_{0}:\left(z_{o}, w_{o}\right)$ can be found which is mapped $o$-homeomorphically by $T_{3}$ onto the interior of a circle in the $w$-plane. This circle can then be mapped one-to-one and conformally onto the unit circle: $|w|<1$, and thus coordinates in the unit circle are assigned to a neighborhood of each $P_{o}$. If two such neighborhoods overlap, the maps $T_{3}$ coincide on the common part and hence the resulting transformation of coordinates in the unit circle is conformal. Thus $R$ is a Riemann surface and $T_{3}$ is a conformal map of $R$ into the $w$-plane. $R$ can thus be thought of as "lying over the $w$-plane" and one can discuss the "sheets of $R$ " over a given point in the $w$-plane.

REMARK. The mapping $T_{1}$ is "interior" in the sense of Stoillow and the construction of the topologically equivalent map $T_{3}$, as just described, is essentially the same as Stoillow's construction of an analytic function topologically equivalent to an interior transformation of a surface into a sphere. (Cf. [6], especially chap. $\mathrm{V}, \S 5$.)

2.4. The family $\Phi$ on the Riemann surface $R$. The homeomorphic transformation $T_{2}$ takes $F$ onto a regular curve-family $\Phi$ filling $R$. In fact, $\Phi$ consists precisely of the "parallel lines" in $R$ lying above the lines $u=$ constant in the $w$-plane. Thus $\Phi$ might be described as a family of parallel lines filling $R$. In a sense, it has thus been shown that the curve-family $F$, assumed to be only locally homeomorphic to parallel lines, is actually equivalent to a family of parallel lines in the large.

The family $G$ of orthogonal trajectories is mapped onto a similar family $\Gamma$ of "parallel lines" filling $R$, all perpendicular to those of $\Phi$. The family $G$ is of no further interest here. However, a question of definite significance is that of the relationships between the topological structures of $F$ and $G$. $F$ may have the structure of a family of parallel lines filling the plane, while $G$ does not, as simple examples show. 
2.5. Conclusion of proof of Theorem 1. To complete the proof, it is simply necessary to apply the fundamental theorem of conformal mapping to $R$. $R$ is homeomorphic to the finite $z$-plane, is hence open and simply-connected. Therefore $R$ can be mapped one-to-one and conformally onto either $D_{1}:|z|<1$ or $D_{\infty}:|z|<\infty$. Let $T_{4}: z \rightarrow P(z, w)$ be the inverse mapping from $D_{1}$ or $D_{\infty}$ to $R$. Then the map $T_{5}=T_{3} T_{4}$ is a conformal map of the domain $D_{1}$ or $D_{\infty}$ into the $w$-plane. Let $H$ be the counter-image of the family $\Phi$ under $T_{4}$. Then under $T_{5}, H$ is mapped onto a family of lines $u=$ constant in the $w$-plane; that is, $H$ is precisely the locus of curves $u(z)=$ const., $u$ being now a harmonic function. But $H=T_{2} T_{4}^{-1}(F)$ is $o$-equivalent to $F$. Hence $F$ is $o$-equivalent to the level-curve family of a function $u(z)$ harmonic in a domain $D$.

\section{NoRmal SUbDivisions of CURVE-FAMILIES AND RIEMANN SURFACES}

3.1. Introductory remarks. Throughout this section $D$ will denote a simply-connected domain and $w=\phi(z)$ a function analytic in $D$ with nonvanishing derivative in $D$. The goal of this section is then to establish Theorem 2 , which describes certain properties of the Riemann surface $R$ of the inverse of $\phi(z)$.

Any statement about $R$ can be restated as a property of the mapping from $D$ to the $w$-plane by $\phi(z)$. Since only topological properties of $R$ are involved, the statements could then equally well be applied to any mapping topologically equivalent to $\phi(z)$, for example, to the mapping $T_{1}$ of $\S 2$, which one might described as "a homeomorphism generated by a regular curve-family filling the plane," or, equivalently, to any locally one-to-one interior transformation (in the sense of Stoillow) of $D$ into the $w$-plane. In all cases precisely the same class of Riemann surfaces $R$ (as constructed in \$2.3) are involved, and there is therefore no loss of generality in considering $R$ as the Riemann surface of the inverse of an analytic function.

3.2. Normal subdivision of a curve-family. The concept of normal subdivision of a regular curve-family $F$ filling the plane is defined in $[1, \S 3]$. The essential ideas will be briefly recalled here, with minor modifications. The normal subdivision consists in the selection of a countable class $F_{\alpha}$ of nonoverlapping sub-families, where $\alpha$ ranges over a countable class $A$ (containing at least one element $\alpha_{0}$ ) and the $F_{\alpha}$ satisfy the following conditions:

1. $F_{\alpha_{0}}$ is equivalent to a family of parallel lines filling a plane; for $\alpha \neq \alpha_{0}$, $F_{\alpha}$ is equivalent to a family of parallel lines filling a closed half-plane, the curve corresponding to the boundary of the half-plane being denoted by $C_{\alpha}$.

2. The common boundary of two sets $F_{\alpha_{1}}$ and $F_{\alpha_{2}}$, if nonvoid, consists of one of the two curves $C_{\alpha_{1}}, C_{\alpha_{2}}$.

3. If $C_{\alpha_{2}}$ is the common boundary of $F_{\alpha_{1}}$ and $F_{\alpha_{2}}$, then there exist curves $C^{\prime}$ and $C^{\prime \prime}$ of the family $F_{\alpha_{1}}$ such that no one of the curves $C^{\prime}, C^{\prime \prime}, C_{\alpha_{2}}$ separates the other two.

3.3. Proof of Theorem 2. The family $F$ of level curves of $u=\operatorname{Re}[\phi(z)]$ 
is a regular cuve-family filling $D$. Since $D$ is homeomorphic to a plane, all the topological properties of regular curve-families filling the plane hold without modification for $F$. In particular, since $F$ is differentiable, the transformation $T_{1}: u=f(x, y), v=g(x, y)$ can be constructed as above, with $u=$ const. on each curve of $F$. In fact, a possible choice of $T_{1}$ is $w=\phi(z)$, and this choice will be made, so that $f=\operatorname{Re}[\phi(z)], g=\operatorname{Im}[\phi(z)]$. The transformations $T_{2}$ and $T_{3}$ can then also be defined as in $\S 2$, and $R$, as defined there, is precisely the Riemann surface of the inverse of $\phi(z)$.

Furthermore, $F$ can be normally subdivided, as in $\$ 3.2$, and a fixed normal subdivision will be assumed. The set $T_{2}\left(F_{\alpha}\right)$ on $R$ will be denoted by $R_{\alpha}$ and its projection $T_{3}\left(R_{\alpha}\right)$ on the $w$-plane by $W_{\alpha}$. The value of $u$ on $C_{\alpha}$ will be denoted by $u_{\alpha}$.

Now the function $u=f(x, y)$ has no local extrema. Since each $F_{\alpha}$ is equivalent to a family of parallel lines, this implies that $u$ takes each value at most once in $F_{\alpha}$ and must range over an interval $I_{\alpha}$ which is open for $\alpha=\alpha_{0}$ and half-open with end points $u_{\alpha}$ otherwise. Since $v$ has no local extrema, $v$ takes each value at most once on each curve $C$ of $F_{\alpha}$, and thus ranges over an interval $p_{\alpha}(u)<v<q_{\alpha}(u)$. It follows that $T_{1}$ is one-to-one in $F_{\alpha}$ and, since $T_{1}$ is locally a homeomorphism, is hence a homeomorphism of $F_{\alpha}$ onto $W_{\alpha}$. Since $T_{2}$ is a homeomorphism, this implies that $T_{3}=T_{1} T_{2}^{-1}$ is a homeomorphism of $R_{\alpha}$ onto $W_{\alpha}$; that is, $R_{\alpha}$ is schlicht over $W_{\alpha}$. Thus (a) is proved.

The common boundary of $R_{\alpha_{1}}$, and $R_{\alpha_{2}}$ (if nonvoid) is the image, under the homeomorphism $T_{2}$, of the common boundary of $F_{\alpha_{1}}$ and $F_{\alpha_{2}}$, that is, of $C_{\alpha_{1}}$ or $C_{\alpha_{2}}$. Since $u=$ const. on $C_{\alpha_{1}}$ or $C_{\alpha_{2}}$, assertion (b) follows.

The representation of $W_{\alpha}$ in the form $p_{\alpha}(u)<v<q_{\alpha}(u), u \in I_{\alpha}$, has already been established in the proof of (a), and (c1) and (c2) also follow immediately. Since $W_{\alpha}$ is a homeomorphic image of $F_{\alpha}$, each point $(u, v)$ of $W_{\alpha}$ for $u$ interior to $I_{\alpha}$ is an interior point of $W_{\alpha}$ (by "invariance of domain"). This implies the indicated semi-continuity of $p_{\alpha}(u)$ and $q_{\alpha}(u)$ for $u$ interior to $I_{\alpha}$. If $u$ is an end point $u_{\alpha}$, then each point $\left(u_{\alpha}, v\right)$ of $W_{\alpha}$ is an image of a point $P$ on $C_{\alpha}$. Since $T_{1}$ is locally a homeomorphism, there exists a semi-circle in $W_{\alpha}$ with center at $\left(u_{\alpha}, v\right)$ and diameter on $u=u_{\alpha}$. This implies the semicontinuity conditions for $u=u_{\alpha}$. Thus (c) is completely proved, and Theorem 2 is established.

REMARK. A further use of the properties of the normal subdivision or of properties of analytic functions would show that the image under $T_{1}$ of each boundary curve $C_{\alpha_{j}}$ of $F_{\alpha}$ lies on one of the two intervals $u=u_{\alpha_{j}}, v>q_{\alpha}\left(u_{\alpha_{j}}\right)$ or $u=u_{\alpha_{j}}, v<p_{\alpha}\left(u_{\alpha_{j}}\right)$. The choice of interval depends on the "side of $F_{\alpha_{1}}$ " to which $C_{\alpha_{j}}$ belongs. Furthermore those curves $C_{\alpha_{j}}$ which map into a fixed one of the two intervals can be grouped into two classes, on each of which $T_{1}$ is a homeomorphism. The two classes are separated by the curve $C$ of $F_{\alpha}$ on which $u=u_{\alpha_{2}}=u_{\alpha_{3}}=\ldots$. The basis for this analysis is given in [1] and [2]. especially $[1, \S 4]$ and $[2, \S 1.4]$. 


\section{Proof of Theorem 3}

In the proof of Theorem 1 above no indication was given as to which type of Riemann surface $R$ might be expected: that is, whether $D$ is $D_{1}$ (hyperbolic case) or $D_{\infty}$ (parabolic case) in the conclusion to Theorem 1. Theorem 3 asserts that $D$ can always be chosen as $D_{1}$ and that there are infinitely many topologically distinct types of $F$ for which $D_{\infty}$ cannot be used.

To prove the first of these assertions, it is necessary only to ensure that $|w|$ be bounded on $R$. The level-curve functions $f(z)$ and $g(z)$ of $\$ 2.2$ can be replaced by the functions

$$
\bar{f}(z)=\tanh f(z), \quad \bar{g}(z)=\tanh g(z)
$$

to ensure that this is the case. For then $\bar{f}$ and $\bar{g}$ are proper level-curve functions, since $\tanh x$ is a monotone continuous function of $x$, and $|\bar{f}|<1$, $|\bar{g}|<1$. Thus $|w|=|\bar{f}(z)+i \bar{g}(z)|$ will be bounded on $R$ and hence $T_{5}$ is a conformal map on $D$ which is bounded. By the Liouville Theorem, $D$ can be $D_{\mathbf{1}}$, but not $D_{\infty}$. Hence every $F$ is hyperbolic.

If an $F$ is also parabolic, then the image family $H$ can be chosen as the level-curve family of the real part of an entire function $\phi(z)$ with nonvanishing derivative. Thus $\phi$ must be either a polynomial of degree one or a function of the form $\int \exp \psi(z) d z$, where $\psi(z)$ is entire. By the Picard Theorem, the Riemann surface $R$ must, in the latter case, have infinitely many sheets. But each sheet of $R$ corresponds to one subset $F_{\alpha}$ of a normal subdivision of $F$, hence each normal subdivision of $F$ must require infinitely many subsets $F_{\alpha}$. In the other case, when $\phi$ is a linear function, $F$ must have the structure of a family of parallel lines. But it is easy to construct examples of families $F$ for which every normal subdivision requires a finite number of subset $F_{\alpha}$. Thus the level-curve families of the functions $f_{n}(x, y)$ defined as follows:

$$
\begin{array}{lrl}
f_{n} & =e^{x} \sin y, & 0 \leqq y \leqq 2 n \pi, \\
f_{n}=y, & -\infty<y \leqq 0, \\
f_{n}=y-2 n \pi, & 2 n \pi \leqq y<\infty,
\end{array}
$$

each require precisely $2 n+1$ subsets $F_{\alpha}$ in every normal subdivision, as can be easily verified. Thus they represent an infinity of distinct topological types of families, none of which can be of parabolic type.

That there are infinitely many topological types of $F$ which are parabolic appears extremely probable, although a demonstration has not as yet been worked out. A preliminary investigation indicates that the level-curve families of the real parts of the entire functions

$$
\exp z, \exp (\exp z), \exp (\exp (\exp z)), \cdots
$$

are all topologically distinct. 


\section{Alternate Development. Unsolved PROBlems}

5.1. Alternate development of the theory. It is of logical interest to consider the most efficient order in which the various theorems occurring in this theory can be established. For convenience, the main theorems are restated here in concise form: I. Possibility of normal subdivision of a regular curvefamily $F$ filling the plane [I, §3.6]; II. Existence of a level-curve function for $F$ (Theorem A above); III. Representation of every "normal chordal system" as a family $F[2, \S 1]$; IV. $o$-equivalence of curve-families having isomorphic chordal systems $[2, \S 2]$; V. Representation of $F$ as a differentiable family (Theorem B above); VI. Equivalence of $F$ with the level-curve family of a harmonic function (Theorem 1 above).

The order of proof thus far is as follows: first I; then II, IV, V as consequences of I, but independently of each other; then VI as a consequence of II and V; then III independently of all others.

It is worth remarking that in the proof of each of the theorems III, IV, V, VI a special canonical model of a regular curve-family was constructed, a different model being used for each theorem. The efficiency of the development would be greatly improved if the number of models were decreased, the ideal being to use just one model.

It has been found possible to proceed as follows: to prove I as before; then to prove III, using as model the family $\Phi$ on the Riemann surface $R$ described above, the regularity of $\Phi$ being immediately verifiable; then to prove IV as before; finally to obtain II, V and VI as immediate corollaries. This method would require two models. However, IV could also be proved with use of the model $\Phi$, although the details have not as yet been worked out. Thus the whole development can be greatly shortened and simplified, and the number of models can be reduced to two, even possibly to one. It is planned to carry out such a program in a subsequent paper.

5.2. Unsolved problems. The results obtained above suggest a number of questions whose answers would be of interest.

1. Are there infinitely many topological types of parabolic families? Are there a continuum? Are there a continuum of families of non-parabolic type?

2. Can the families of parabolic type be characterized in terms of chordal system structure?

3. What function-theoretic properties characterize a class of entire functions whose level-curve families (that is, those of their real parts) have a given topological type?

4. Can Theorem 1 of this paper be extended to regular curve-families with singular points? The singular points would then have to be of general zed saddle-point type (such as that of the level curves of $\operatorname{Re}\left(z^{n}\right)$ at the origin). This question suggests a general program of classification of curve-families with such singular points. 
5. What are the relationships between the structure of the family $F$ and that of its family $G$ of orthogonal trajectories?

The question arises as to whether this theory can be of use in the solution of the problem of type for Riemann surfaces. One is necessarily restricted thus far to Riemann surfaces with no algebraic branch points. The answer to question 2 would give a necessary condition that a family be parabolic; the failure of this condition would then be a sufficient condition that a family be hyperbolic. This result could then be restated in terms of the Riemann surface $R$, and might well give a useful criterion for function theory.

\section{BIBLIOGRAPHY}

1. W. Kaplan, Regular curve-families filling the plane, I, Duke Math. J. vol. 7 (1940) pp. $154-185$.

2. —_, Regular curve-families filling the plane, II, Duke Math. J. vol. 8 (1941) pp. 1146.

3. - The structure of a curve-family on a surface in the neighborhood of an isolated singularity, Amer. J. Math. vol. 64 (1942) pp. 1-35.

4. Differentiability of regular curve-families on the sphere, Lectures in Topology, Ann Arbor, 1941, pp. 299-301.

5. R. Nevanlinna, Eindeutige analytische Funktionen, Berlin, 1936.

6. S. Stollow, Leçons sur les principes topologique de la théorie des fonctions analytiques, Paris, 1938.

7. H. Whitney, Regular families of curves, Ann. of Math. (2) vol. 34 (1933) pp. 244-270.

Additional references are given in [1].

UNIVERSITY OF MICHIGAN,

ANN ARBOR, Mich. 